\title{
Communications for a Picture Archiving Communications System with a Parallel Operating Image Data Base
}

\author{
Gerard L. Reijns and Alfred Kayser
}

In cooperation with BAZIS (Support Group Hospital Information Systems at Leiden, The Netherlands), we started the design of a Picture Archiving and Communications System (PACS) a few years ago that contains a centralized image data base. By means of system modeling and measuring some performance parameters, we have discovered that such a system cannot provide the required response times. This is mainly because of equipment communication interfaces that are not fast enoigh and have a throughput that does not generally exceed 16 Mbits/second. By providing a parallel operating image buffer system, the problems related to the limited speed of the communication interfaces can be overcome. Moreover, by splitting the network into a number of subnets connected to each other by means of "bridges," the communications load can be locally reduced, improving in this manner the response times. Our PACS contains a supervisor and one relational data base that relates patient data with image locations. A significant part of the report is concerned with the design of the high speed American College of Radiology-National Electronic Manufacturing Association transmission control protocol/internet protocol (ACR-NEMA TCP/IP) communications interface. The use of such an interface eliminates the need for a Network Interface Unit, reducing in this manner communication delays and complexity. We have developed and have in operation for experimental purposes a small-scale PACS, consisting of a number of workstations, a relational data base and image buffers, all connected to a network that uses the ACR-NEMA TCP/IP protocols. This experimental PACS is used to provide input data to a PACS performance model, to validate this model, and to investigate alternative system configurations.

Copyright $\odot 1993$ by W.B. Saunders Company

KEY WORDS: PACS, parallel processing, computer communication, TCP/IP, image communication, ACR/ NEMA, communication interface.

$\mathbf{T}$ HE PICTURE Archiving and Communications System (PACS) is an electronic computerized system that will replace the existing film-based system in hospitals within the next few years. PACS has a number of advantages over the present film-based system, such as the capability to display patient images at many locations in a hospital a few seconds after they have been requested. It is also possible to display the same image simultaneously at different locations, enabling exchange of information by means of speech and symbols on the image screen. PACS will also provide a saving in space and personnel for the archiving and retrieving of films. However, despite the many years spent on research, design, and experimentation with small scale PACS systems, its implementation at full scale in hospitals has, with very few exceptions, yet to occur. This delay in implementation at full scale has several reasons.

In the past and even today, a full PACS system is still more expensive than the existing film-based system using alternators for viewing. To become price competitive, the routine production of hard copy photos should be abandonned. This means that $x$-ray and other image acquisition devices must directly generate digital images with the required resolution. Although such $\mathrm{x}$-ray equipment is not yet on the market, prototypes with a resolution of $500 \times$ 500 pixels presently exist. It is expected that systems with a resolution of $1,000 \times 1,000$ will become commercially available within the next few years.

The tremendous amount of image data that has to be stored and speedily communicated requires very advanced technology and careful architectural design. The workstation technology, data storage, and communication capabilities have only recently reached a level that enables the design and implementation of a full PACS with the required performances at acceptable cost figures.

In a PACS, we will find equipment produced by different manufacturers, such as $\mathrm{x}$-ray equipment, ultrasound, tomography, workstations, and storage devices. It is of utmost importance that equipment from different manufacturers can be interconnected and compatible. Such standardization has to be established at different Open Systems Interconnection (OSI) levels, eg, at the data formatting level and at the

From the Faculty of Electrical Engineening, Delft University of Technology, Delft, The Netherlands.

Address reprint requests to G.L. Reijns, Professor of Computer Science, Faculty of Electrical Engineering, Delft University of Technology, Mekelweg 4, PO Box 5031, 2600 GA Delft, The Netherlands.

Copyright 1993 by W.B. Saunders Company

0897-1889/93/0601-0005\$5.00/0 
different communication levels. Standardization of the data formatting has partly been obtained (American College of Radiology/ National Electronic Manufacturing Association [ACR/NEMA]), but the necessary high speed interfaces are lacking. More standards must be agreed on.

Last but not least, working in a PACS environment is quite different from working with the present film-based systems. This requires adaptation of hospital personnel, training, and reorganization.

In particular, the rapid availability of PACS images in a hospital is a great advantage with respect to the present film-based system in that the film-based system sometimes takes hours to retrieve images and have them delivered to the right locations.

\section{CENTRAL IMAGE BASE VERSUS A DISTRIBUTED IMAGE BASE}

We have studied a PACS with a centralized image data base (Fig 1) $)^{1-4}$ in cooperation with Bazis (Support Group Hospital Information Systems) at Leiden, The Netherlands. To provide a system with an acceptable response time, we divided the storage system into three hierarchical levels: (1) a very large (a few hundred Gbyte) but very slow optical disk archive with an access time of approximately 10 seconds; (2) a much smaller (a few Gbyte) buffer at intermediate level, sufficient to store the patient data, which is handled during one particular day in a hospital (the access time of this magnetic disk store is approximately 20 milliseconds); and (3) a relatively small image memory at each of the

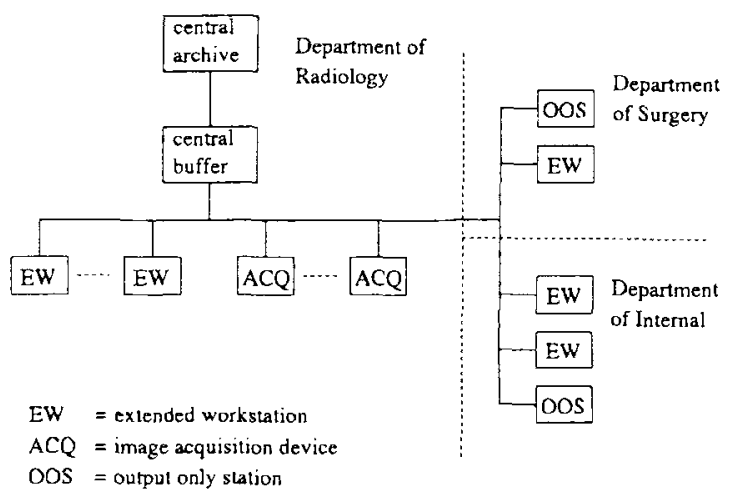

Fig 1. PACS with a centralized image base buffers.

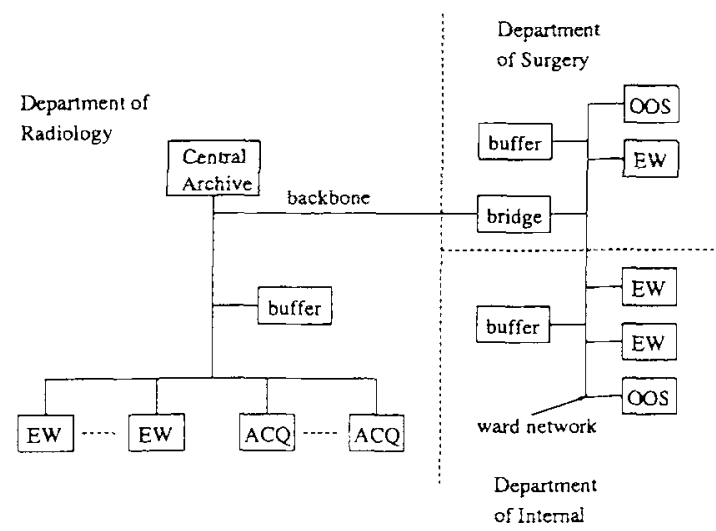

Fig 2. PACS with distributed image base buffers.

workstations, able to store the sets of images of one or two patients.

In addition to the department of radiology, all wards in a hospital that handle images were planned to receive the requested images through the centralized buffer in this configuration of Fig 1. In our design, this buffer at the intermediate level consisted of a mainframe with many magnetic disks. Performance modeling and some preliminary measurements ${ }^{2}$ have shown that such a system could not provide the required response times, one of the bottlenecks being the communication interface of the mainframe. Moreover, the mainframe solution was quite an expensive solution. It also became apparent that image data, which is frequently consulted in a particular ward, can preferably remain stored in that ward, for instance in the department of surgery.

For these reasons, the design was changed and the centralized buffer in Fig 1 was replaced by distributed image base buffers (Fig 2). ${ }^{5}$ At the same time, a communications bridge was introduced. This bridge splits the communication network into two parts: one part, connects the different units in the department of radiology and another part connects the workstations of all other wards. The communication traffic, which remains locally within the department of radiology, does not pass the bridge. On the other hand, the traffic on the ward network is not "seen" by the backbone as long as no references are made to data in the department of radiology. However, if an image is requested from a workstation in a ward and cannot be found in the ward buffer, the image must be 
fetched from the central archive or from a buffer in the department of radiology and the bridge then enables the transfer of data between the two parts of the network.

Therefore, the installation of a bridge will reduce the amount of traffic on both parts of the network resulting in improved response times. If the amount of traffic on the ward network would also become excessive, more bridges may be installed to split the total number of wards into sections. In certain circumstances it would even be justified to provide the wards with their own archive, reducing even further the amount of traffic via the bridge.

\section{PACS WITH A PARALLEL IMAGE DATA BASE}

Our further discussion on PACS will now be focussed on that part of the PACS which will be used in the department of radiology or in another important department, such as internal or surgery. This means that we will not consider here important issues related to the communications between the wards and the department of radiology.

Figure 3 shows the present PACS configuration for the department of radiology. The archive will contain the large image data base and

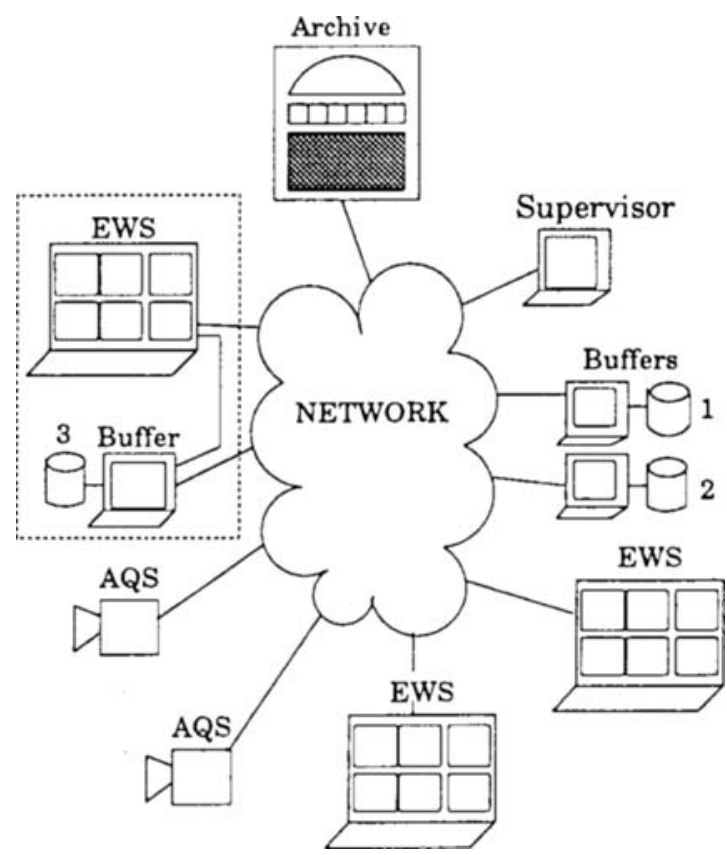

Fig 3. A PACS with parallel operating image buffers in a radiology department. will consist of a jukebox filled with optical disks that will contain the images to be used for a period of 100 days or more. Because the archive has a long access time (approximately $10 \mathrm{sec}-$ onds), a subset of images, which are going to be used on the current day, is stored in a buffer. A more detailed investigation of the department of radiology, taking actual workloads into account, revealed that the one-day image buffer at intermediate level needed to consist of a number of parallel operating buffers to obtain the required response times.

As shown in previous reports, ${ }^{2,6}$ a system throughput with a maximum rate of approximately $50 \mathrm{Mbits} / \mathrm{sec}$ is required to reach acceptable response times of 2 to 3 seconds. Because we and others 7.8 have not succeeded in implementing a communication interface with a speed higher than $16 \mathrm{Mbits} / \mathrm{sec}$, we were looking for system parallelism as a solution. We have found this solution by storing the images not in one buffer at intermediate level but in a parallel operating multibuffer, consisting of the computers 1, 2 and 3 in Fig 3. In doing so, we solved simultaneously another problem which is inherent in the one-buffer system, ie, that of the restricted disk data rate of approximately 16 Mbits/sec. Another important advantage of a multibuffer image base solution will be its potential for fault tolerancy.

Last but not least, we believe that a system using a number of large workstations for the implementation of an image data base at intermediate level will be more cost effective than using a mainframe for this purpose. The different units in Fig 3, eg, archive, buffers, workstations, and acquisition stations are all connected to a 100 -Mbits/sec optical fiber network using the fiber distributed data interface (FDDI) communications protocol. The FDDI bit rate can be fully exploited by means of time multiplexing the communications of the different connected units.

One computer, called the supervisor, controls the system of Fig 3 and also incorporates the patient data base. Before accessing the image base, this patient data base must first be queried to find the relations between the patient identifiers and the locations of the corresponding patient images. With the images stored in several computers, the relational data base can be 
either of the centralized type, eg, all workstations in the department of radiology query only one relational data base or are of the distributed type. Updating the data on a distributed relational data base requires careful design to keep the data coherent. We have chosen a central relational data base, avoiding the data coherency problem. However, this solution may cause performance and reliability problems for which we must find a solution in future.

Figure 4 shows the data flow. (1) When a user wants to monitor a set of images from a patient, he/she sends a query to the supervisor. (2) The supervisor queries the structured query language (SQL) data base to find the location of the images. Subsequently, the supervisor asks either one of the bufferstations or the archive to send the data (images) to the user (4). Physically, the supervisor and SQL data base can be implemented in one computer.

\section{INTEGRATION OF BUFFERS AND WORKSTATIONS IN A PARALLEL SYSTEM}

Why are there still separate buffers 1,2, 3 and is it possible to integrate these buffers with the extended workstations? Disk capacity has now become very inexpensive and a workstation can be easily equipped with 1 Gbyte of disk memory or more. The answer is that, although present workstations are very fast, it is not unlikely that they are still too slow to execute both the function of buffer and that of extended workstation and meet the performance requirements. This will depend very much on the specific applications that will run on an extended workstation. Nevertheless, there are obvious advantages to combine these functions in one com-

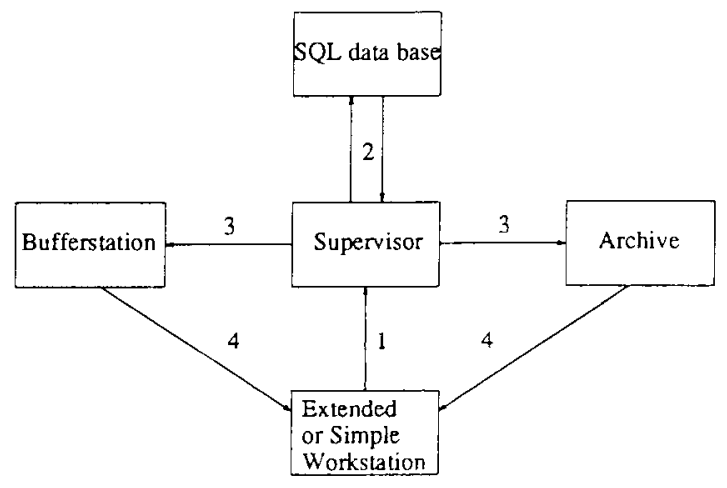

Fig 4. Data flow. puter, because a certain percentage of images which the workstation will call for display will already be present in the same computer. The ideal situation would be one in which the bulk of the images that are required at a workstation could be preloaded in this station; however, operationally this will not always be possible. For that reason we are experimenting with a solution as given in Fig 3, in which one of the image buffers (number 3 ) is colocated with a workstation. Direct communication of images between computer 3 and the extended workstation, bypassing the Image Network in that manner, will be made available so that the load on the Network may be reduced.

\section{ACR-NEMA COMMANDS IN A PARALLEL OPERATING IMAGE DATA BASE ENVIRONMENT}

ACR-NEMA has defined a number of command-requests and command-responses at application level. These are: SEND, FIND, GET, MOVE, DIALOG, CANCEL, and ECHO. ${ }^{9}$ These commands have probably been defined without considering a parallel operating image data base implementation with separate supervisor. This caused some problems for our data base version which are discussed below.

Suppose a new image has been acquired and it must be stored in the image data base. The supervisor has the knowledge to instruct the system where the acquired image should be stored. In a centralized data base system, the SEND_REQUEST would be used by the acquisition station to send the image to the data base. In this parallel operating image data base solution, the SEND_REQUEST command cannot be used because the destination address is not known by the acquisition station. We have solved this problem by having the acquisition station first send a FIND_REQUEST command with a shadow group to the supervisor. The shadow group informs the supervisor that this FIND command must get special treatment and that a new image must be stored somewhere in the data base. After having sent a FIND_RESPONSE as confirmation, the supervisor transmits a MOVE_REQUEST command to the acquisition station to direct the data to the selected destination. Although the use of a special FIND_REQUEST command with a 
shadow group is an ACR/NEMA compatible method, it is not an appropriate way of handling this problem.

A similar problem exists when a workstation must retrieve an image or a set of images for the image data base. The GET command cannot be used because the location where the image can be found is not known to the workstation. We solved this problem by having the workstation send a special FIND_REQUEST command to the supervisor, which in turn will send a MOVE_REQUEST command to the specific buffer to have the data transferred to the workstation. Again, this is a somewhat improper use of the FIND command, although not incorrect.

\section{ACR/NEMA-TRANSMISSION CONTROL/INTERNET PROTOCOLS}

\section{General}

The ACR/NEMA communication standard, which was developed for the communication of digital medical images, corresponds to the OSI reference model. The ACR/NEMA target data rate at the physical level is $8 \mathrm{Mbytes} / \mathrm{sec}$. Figure 5 shows a comparison between the OSI model and ACR/NEMA layers.

The ACR/NEMA interface does not have network capabilities. The equipment provided with an ACR/NEMA interface can communicate with only one other apparatus that is equipped with such an interface (point-to-point communication). If images must be communicated to various equipment via a network, the ACR/NEMA interface must be connected to a network interface unit (NIU), which in turn is connected to the network (Fig 6).
OSI

\begin{tabular}{|l|}
\hline Application \\
\hline Presentation \\
\hline Session \\
\hline Transport \\
\hline Network \\
\hline Datalink \\
\hline Physical \\
\hline
\end{tabular}

ACR-NEMA

\begin{tabular}{|l|}
\hline Application \\
\hline Presentation \\
\hline Session \\
\hline Transport \\
\hline Datalink \\
\hline Physical \\
\hline
\end{tabular}

Fig 5. Comparison between OSI and ACR/NEMA.

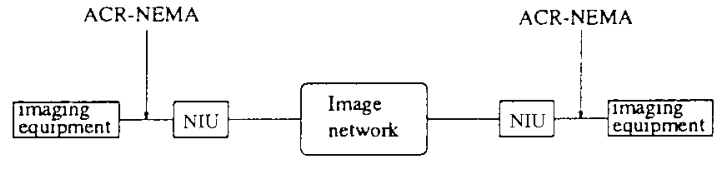

Fig 6. Network with NIUs.

Figure 7 shows in more detail the OSI layers in the imaging equipment and the NIU. The message handling performed in session, transport, datalink, and physical layer of the imaging equipment interface must be undone again in the NIU. The NIU shown in Fig 7 has a transmission control protocol/internet protocol interface connected to a FDDI for optical fiber communication. Instead of the FDDI, ethernet can be used, but in general this would be too slow for PACS.

To simplify the interfacing and improve the performance, we have designed and built an interface with layers as indicated in Fig 8. Before discussing this design in more detail, an overview is given of the operations in each of the layers of Fig 8 .

\section{Definition of the Different ACR-NEMA TCP/IP Layers}

Application layer. ACR/NEMA defines a number of commands that can be used to locate and move data. At this level, a minimum number of commands are defined: SEND, FIND, GET, MOVE, DIALOG, CANCEL and ECHO. The emitting side of these commands will issue command requests, and the receiving side will reply by one or more command responses. Most of the command messages are short, typically less than 250 bytes. However, the SEND_REQUEST carries data and can contain several millions of bytes.

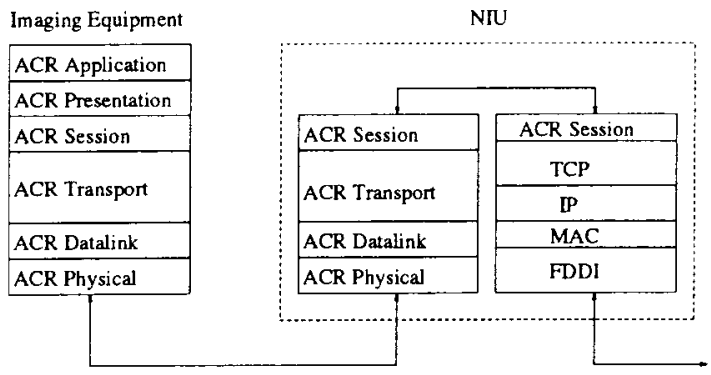

Fig 7. The NIU in detail. 


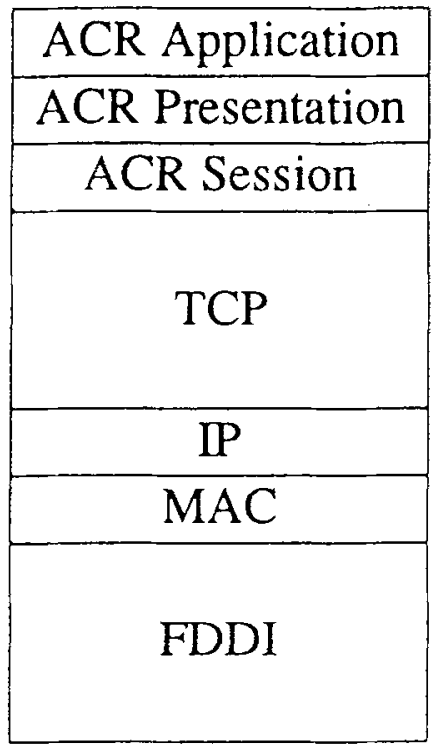

Fig 8. The designed interface.

Presentation layer. All commands passed by the application layer to the presentation layer are formatted into a well defined structure. Each message is organized into numbered groups representing the general categories of information. There are a number of standard groups defined. The groups have a fixed numbering and a message must be transmitted in ascending order of group numbers. A message consists of a command group (number 0000) followed by groups of the data type. Each group is subdivided into data elements. The components of a data element are contained in four fields: a group number, a data element number, a length, and a value. When storing information, the groups of a message containing the image and related patient information should preferably be stored in the ACR/NEMA format. Only the command group preceding these data groups must then be assembled in real time when a message is going to be transmitted.

Session layer. The session layer takes care of opening and closing the necessary logical channels using the transport layer beneath it. To do this it must bind logical names to network addresses by maintaining a list of addresses of all other stations. Furthermore, it maintains a queue of information about the processed messages and takes care of retransmitting failed messages.

TCP. The TCP is a connection-oriented, end-to-end reliable protocol designed to fit into a layered hierarchy of protocols that support multinetwork applications. It provides a highly reliable station-to-station protocol. It uses the underlying transport mechanism, for example IP datagrams, to transmit its packets. TCP recovers data that is damaged, lost, duplicated, or delivered out of order by the underlying communication system. This is achieved by assigning a sequence number to each packet and requiring a positive acknowledgement from the receiving TCP. If the acknowledgement is not received within a timeout interval, the data is retransmitted. ${ }^{10}$

$I P$. The IP provides for transmitting blocks of data, called datagrams, from sources to destinations, where sources and destinations are stations identified by fixed length addresses. IP also provides for fragmentation and reassembling of long packages. It sends datagrams of variable size, depending on the underlying layers and the provided TCP packages. The header checksum provides a verification that the information in the datagram has been transmitted correctly. If the header section fails, the internet datagram is discarded. ${ }^{11}$

$M A C$ layer. This MAC layer decouples the general TCP/IP software from the specific underlying hardware. ${ }^{12-14}$

Datalink/Physical layer. The datalink layer handles packet interpretation, packet framing, and flow control. The physical layer is concerned with the transmitting of individual bits over a communication channel. Examples of datalink/physical layers named in this report are FDDI and Ethernet. The FDDI defines a standard for optical fiber token ring networks. It defines a speed of $100 \mathrm{Mbits} / \mathrm{sec}$ over a distance up to 2 kilometers. ${ }^{15,16}$ Ethernet defines a carrier sense multiple access network with collision detection (CSMA/CD) with speeds up to 10 $\mathrm{Mbits} / \mathrm{sec}$

\section{SOME PERFORMANCE MEASUREMENTS}

Before starting the design of the ACRNEMA TCP/IP interface, we have investigated the feasibility of using TCP/IP in a high-speed PACS environment. The performance of network communications depends largely on the delays of the transmission medium and the communication interfaces. Some well-known transmission media are Ethernet and FDDI. Ethernet is a one-persistent CSMA/CD proto- 
Table 1. Some Measurements of TCP/IP

\begin{tabular}{ccrl}
\hline Source & Sink & Kbytes/sec & \multicolumn{1}{c}{ Comments } \\
\hline Sparc2 & RS6000 & 600 & Ethernet \\
Sparc2 & Sparc2 & 2300 & Local loopback \\
RS6000 & RS6000 & 1000 & Local loopback \\
\hline
\end{tabular}

col over a coax bus network and usually has a transmission speed of $10 \mathrm{Mbits} / \mathrm{s}$. FDDI uses an optical fiber as its medium, and its protocol is based on the token ring access method. The transmission speed of FDDI is $100 \mathrm{Mbits} / \mathrm{s}$. Of the communication interface, the TCP/IP delays are difficult to analyse, particularly in combination with the lower transmission layer ${ }^{17}$ To predict the behavior, some measurements were done on the throughput of TCP/IP on ethernet and via the loopback interface (Table 1). The loopback interface is used when source and destination are the same machine. This is comparable with TCP/IP without a transmission layer.

Table 1 presents some numbers concerning TCP/IP performance. These numbers were obtained using a TCP/IP source and sink application, under the condition that no other applications were running on the used machines. Of course the execution time of the TCP/IP code depends on the kind of central processing unit (CPU), memory bus bandwidth, and the percentage of time the CPU can spend on the communication application. It is estimated that using Sparc2 stations connected to an FDDI network, a maximum speed of approximately 2 Mbyte/ sec can be reached. The fact that FDDI uses packet sizes up to 4,000 bytes, whereas the maximum packet size in ethernet is 1,460 bytes, reduces the code overhead per byte in respect to ethernet. An FDDI network can transport 12.5 Mbytes/sec. Therefore, by time multiplexing messages on the network and by exploiting the parallelism of the multibuffer, one is able to reach the desired system throughput of 5 to 8 Mbytes/sec.

\section{STRUCTURE OF THE ACR-NEMA TCP/IP INTERFACE SOFTWARE}

\section{Software Requirements}

It can be stated that the PACS software must comply to the ACR-NEMA specifications, use the standard TCP/IP libraries, work with both UNIX and OS/2 operating systems, be easy to use when writing new applications, and have good response times.

\section{Implementation Considerations}

The requirement that it must be easy to write new applications led to the development of a separate universal interface program, because all possible ACR-NEMA stations must have the same communication functions. Using this interface for handling the communication functions, ACR-NEMA applications can be built (namely, application for workstation, application for archive, application for acquisition station).

This separation of the communication functions from the application also resulted in satisfying the requirement that a user program should not have to wait for the communication part of the software while it is handling a message of several megabytes. In Fig 9 the software architecture for a workstation is shown.

In the following paragraphs a general overview of the functions of the interface will be given. The communication between the application and the interface will also be described, followed by a detailed discussion of the interface program.

\section{The Interface Functions}

The interface program must take care of sending images to and receiving images from other stations. When an application wants to send a message, the interface program constructs a command group and links this data

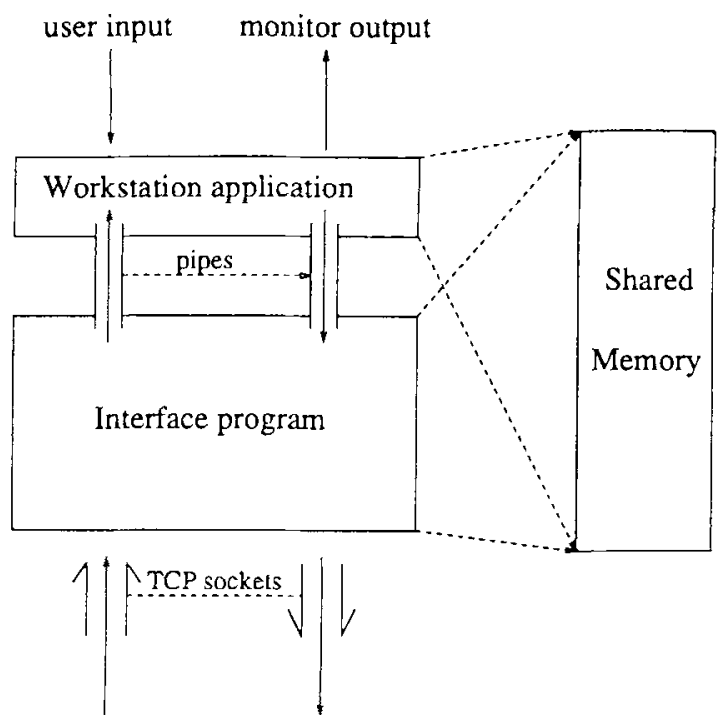

Fig 9. Workstation with communication interface. 
part to the message part provided by the application.

The interface maintains a queue consisting of the send messages. In this queue, the current state of the message is stored and updated until a final reply has been received. Thereafter, a signal will be given to the application program. The interface also links the ACR/NEMA stations names such as "Doctor Ben Johnson" or "Camera 1" to their internet addresses.

Furthermore, the interface program must have the capability to send error responses on request from other stations. This is necessary when, for instance, the application program is out of order or no memory is available to store incoming messages. Besides these obvious reasons, the interface program also checks the message for having a correct ACR/NEMA format, which might result in sending an error response.

\section{The Application Functions}

Using the interface and its functions described above, applications can be written that perform, for instance, the user-computer data exchange, the data base routines, and the supervisor functions.

The application must build a complete message in the ACR/NEMA presentation format (except the command group). This data can be the image generated by a camera or the comment on a certain set of images. In case of a data base application, the application should handle the information retrieval and storage.

\section{Communication Between Application and Interface}

The communication between the application program and the interface program is based on pipes between these programs and pieces of shared memory accessible by both programs. Because copying the data in a different memory part would cause a significant performance loss, the usage of shared memory is chosen. The information that passes through the pipes are buffer identifiers referring to data structures in the shared memory. In these data structures all information needed by both programs and additional information about the status of the segments can be stored.

\section{The Structure of the Interface Program}

Because it is preferable for the interface program to continue its functions while receiving or sending a message of several megabytes, the interface program itself has been split into three processes. The main process handles most of the functions described earlier. The second process, called the send process, creates a TCP socket and writes to it. The third process, a receive process, reads the $T C P$ receive socket. The communication between the main process and the send and receive processes also uses pipes and the same shared memory. This structure of the software for a data base application is shown in Fig 10.

An additional advantage to using separate processes is for the potential of implementing additional send and receive processes, using the same pipes to enable multiple messages to be concurrently sent or received. If an option to send high priority messages is desired, this can be implemented by a separate send process with its own pipes to the interface.

\section{Integration of $A C R-N E M A$ with TCP/IP}

Although TCP is a connection oriented protocol, the interface program uses it as a semiconnectionless transport medium. The receive part initially opens a receive socket when starting up. When receiving a connect request, the TCP software creates a new socket to receive the incoming message. After receiving the entire message, the new socket is closed.

The send process opens a new socket for every message to be sent, using the internet address it gets from the interface. The message is then split by TCP into smaller packets and

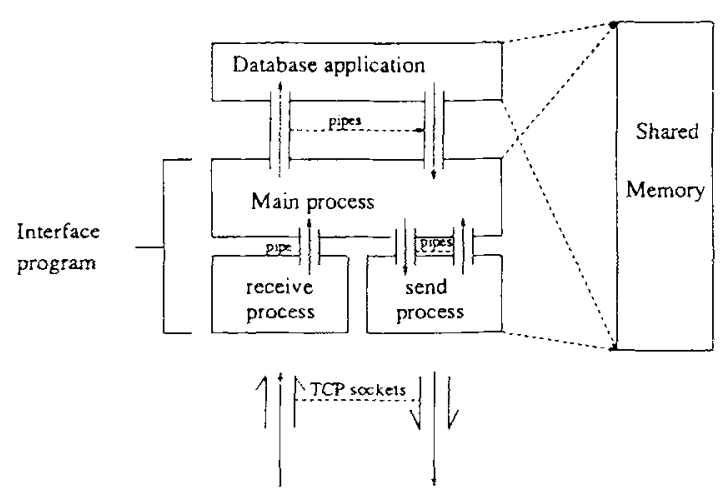

Fig 10. Example of a data base application with the send and receive processes separated. 
sent using IP datagrams. The size of these packets is determined by the TCP layer, depending on the underlying communication layers and the TCP buffer size. After having sent the message, the socket is closed again. The data stream is checked at two levels. The first level is executed by TCP and it checks whether or not the connection oriented point-to-point protocol is reliable. The second level is embedded in the interface program to check responses on correctness and to verify the ACR/NEMA structure of the messages.

\section{CONCLUSIONS}

A PACS design is presented with a large central image store (jukebox with optical disks, access time approximately 10 seconds) and much smaller parallel operating image buffers in which the images are stored that are handled during the current day. The parallel operating image buffers have disks with an access time of approximately 20 milliseconds. Together with the developed fast communication interfaces, they provide the required system response time.

To avoid problems in data coherence, the system has been equipped with a central relational data base that relates patient data with image storage locations. In addition to modeling the system, a small scale laboratory PACS has been developed that enables us to carry out performance measurements on alternative configurations. It is recognized that the central relational data base could become a perfor- mance and reliability bottleneck. In the future, not only the image base, but also the relational data base may need to be distributed.

Presently, we have a laboratory PACS in operation that consists of several (different) types of workstations, a supervisor that also contains the relational data base and several buffer stations, and all are connected through ACR/NEMA TCP/IP protocols to ethernet.

The use of an ACR/NEMA TCP/IP interface whose design is discussed in this report, eliminates the need for separate NIUs reducing communication delays and complexity. In this communication interface design, much attention has been given to optimizing the speed by avoiding, as much as possible, copying actions and running processes in parallel. The design of the ACR/NEMA TCP/IP interface allows an average throughput of 2 Mbyte/sec on a modern workstation and uses an FDDI communication connection. The rate at the FDDI physical layer will be $12.5 \mathrm{Mbyte} / \mathrm{sec}$, which can be fully exploited by multiplexing the communications of the different connected units, each having a lower throughput. Our ACR/NEMA TCP/IP interface operates both under UNIX and the $\mathrm{OS} / 2$ operating system.

\section{ACKNOWLEDGMENT}

We would like to acknowledge the Bazis Group in Leiden and, in particular, Prof A.R. Bakker, Dr F.P. Ottes and W.J.J. Stut for their support in this project, and from our own laboratory, J.A. Haantjes and A.M.A. Stehouwer.

\section{REFERENCES}

1. Massar ADA, de Valk JPJ, Reijns GL, et al: Simulation of an image network in a medical image information system. Proceedings Second International Symposium on Optical and Electro-Optical Applied Science and Engineering, Cannes, November 1985

2. Reijns GL, de Valk JPJ, Bakker AR: Some Simulation and Communication Aspects of PACS. Proceedings SPIE Medical Imaging II Conference, Newport Beach, January 1988

3. Bakker AR, Stut WJJ, de Valk JPJ, et al: Cost Modeling of PACS. Proceedings SPIE Medical Imaging III Conference, Newport Beach, January 29-February 3, 1989

4. Houtekamer GE, Franken LJN, Reijns GL, et al: A Design for a PACS Central Storage. Europacs Conference, Trieste, May 2-4, 1990

5. Kayser A, Reijns GL: A Distributed Data Base for a PACS, Proceedings SPIE, Medical Imaging VI Conference, Newport Beach, February 1992

6. Maydell UM: Medical Image Delays with the ACRNEMA Standard. IEEE Engineering Medicine and Biol Society 11 th Annual International Conference C, 1989
7. McNeill KM, Osada M, Martinez R, et al: Evaluation of the ACR-NEMA Standard for Communications in Digital Radiology. IEEE Trans Med Imaging 9:281-290, 1990

8. Dejarnette WT: Two-chip ACR-NEMA Datalink/ Physical layer implementation proceedings SPIE Medical Imaging IV Conference, Newport Beach, February 6-9, 1990

9. ACR-NEMA: Digital Imaging and Communications Standard. Publication \#300-1988, NEMA, 2101 L St NW, Suite 300, Washington DC 20037, 1988

10. RFC 793: Transmission Control Protocol. DARPA Internet Program Protocol Specification, September 1981

11. RFC 791: Internet Protocol. DARPA Internet Program Protocol Specification, September 1981

12. Postel J, Reynolds J: RFC 1042: A Standard for the Transmission of IP Datagrams over IEEE 802 Networks

13. Katz D: RFC 1103: A Proposed Standard for the Transmission of IP Datagrams over FDDI Networks. June 1989

14. Katz D: RFC 1188: A Proposed Standard for the 
Transmission of IP Datagrams over FDDI Networks, October 1990

15. Gahan C: FDDI, Business Communications for the 1990's. BICC Data Networks 1989, BICC/5K/8/89

16. Ross FE: An Overview of FDDI: The Fiber Distrib- uted Data Interface. IEEE J Selected Areas Communic 7:1043-1051, 1989

17. Clark DD, van Jacobsen, Romkey $\mathbf{J}$, et al: An Analysis of TCP Processing Overhead. IEEE Communications Magazine, June 1989 